\title{
Influence of the Hall term on KH instability and reconnection inside KH vortices
}

\author{
K. Nykyri ${ }^{1,2}$ and A. Otto ${ }^{2}$ \\ ${ }^{1}$ The Blackett Laboratory, Imperial College, London, UK \\ ${ }^{2}$ Geophysical Institute, University of Alaska, Fairbanks, USA
}

Received: 2 February 2003 - Revised: 8 July 2003 - Accepted: 22 July 2003 - Published: 19 March 2004

\begin{abstract}
The Kelvin-Helmholtz instability (KHI) in its nonlinear stage can develop small-scale filamentary field and current structures at the flank boundaries of the magnetosphere. It has been shown previously with MHD simulations that magnetic reconnection can occur inside these narrow current layers, resulting in plasma transport from the solar wind into the magnetosphere. During periods of northward IMF, this transport is sufficient to generate a cold, dense plasma sheet on time scales consistent with satellite observations. However, when the length scales of these narrow current layers approach the ion inertia scale, the MHD approximation is not valid anymore and the Hall term in the Ohm's law must be included. We will study the influence of the Hall term on the KHI with 2-D Hall-MHD simulations and compare our results with corresponding MHD simulations. We estimate plasma transport velocities of the order of $\sim 1.5 \mathrm{~km} / \mathrm{s}$, thus confirming the results of the MHD approximation. However, the fine structure and the growth rates differ from the MHD approximation in an interesting way.
\end{abstract}

Key words. Magnetospheric physics (magnetopause, cusp and boundary layers; plasma waves and instabilities), Space plasma physics (transport processes; magnetic reconnection; numerical simulation studies; nonlinear phenomena; turbulence)

\section{Introduction}

The Kelvin-Helmholtz instability (KHI) has been widely studied both with analytical theory (Chandrasekhar, 1961) and with numerous computer simulations using MHD (Miura and Pritchett, 1982; Miura, 1984, 1987, 1992; Chen et al., 1997; Otto and Fairfield, 2000; Keller and Lysak, 1999; Nykyri and Otto, 2001; Brackbill and Knoll, 2001), HallMHD (Huba, 1994, 1996; Fujimoto and Terasawa, 1991), and hybrid and kinetic codes (Terasawa et al., 1992; Thomas

Correspondence to: K. Nykyri

(k.nykyri@ic.ac.uk) and Winske, 1993; Fujimoto and Terasawa, 1994, 1995; Thomas, 1995). There are also several ionospheric and ground observations (Lee and Olson, 1980; Olson and Rostoker, 1978; Olson, 1986; Ohtani et al., 1999) associated with the KHI, as well as in-situ satellite observations (Fairfield et al., 2000; Nikutowski et al., 2000; Nykyri et al., 2003; Nykyri, 2002), both from dawn and dusk-side flanks of the low-latitude boundary layer. The main objective of the simulation studies of the KHI has been to understand the nonlinear evolution of the $\mathrm{KH}$ mode at the magnetopause and its effect on the momentum and energy transport. Hybrid and full particle simulations also indicate that fast anomalous diffusion is possible for sufficiently thin boundaries in the KH vortex (Fujimoto and Terasawa, 1994, 1995; Thomas and Winske, 1993).

Except for diffusion, the KHI - as an ideal plasma instability - has not traditionally been expected to produce a significant mass transport. However, MHD simulations by Otto and Fairfield (2000) -motivated by the Geotail observations of the KHI (Fairfield et al., 2000)- indicated that reconnection can occur inside the narrow current layers generated by the Kelvin-Helmholtz instability. Nykyri and Otto (2001) quantified this reconnection process inside $\mathrm{KH}$ vortices in two dimensions using MHD approximation. Their results indicate that reconnection inside $\mathrm{KH}$ vortices can transport plasma of solar wind origin into the magnetosphere with a transport velocity of $\sim 1.5 \mathrm{~km} / \mathrm{s}$. This corresponds to a diffusion coefficient of the order of $10^{9} \mathrm{~m}^{2} / \mathrm{s}$, which is sufficient in producing the LLBL during northward IMF conditions on observed time scales (Fujimoto et al., 1998). Brackbill and Knoll (2001) used 3-D MHD simulations in an idealized perpendicular configuration, finding that KHI could cause reconnection on the KH time scale. The MHD approximation assumes large temporal and spatial scales. When the thickness of the boundary layer approaches the ion inertia scale, $c / \omega_{p i}$, the Hall term in generalized Ohm's law becomes important. By including the Hall term, $\boldsymbol{J} \times \boldsymbol{B}$, in the system of equations describing plasma dynamics, one can show that the magnetic field becomes frozen into the 
Table 1. Simulation Normalization.

\begin{tabular}{ll}
\hline Magnetic field $B_{0}$ & $16 \mathrm{nT}$ \\
Number density $n_{0}$ & $11 \mathrm{~cm}^{-3}$ \\
Current density & $22 \mathrm{nA} \mathrm{m}^{-2}$ \\
Length scale $L_{0}$ & $100 \mathrm{~km}^{-1}$ \\
Velocity $v_{A}$ & $105 \mathrm{~km} \mathrm{~s}^{-1}$ \\
Time $\tau_{A}$ & $0.95 \mathrm{~s}$ \\
\hline
\end{tabular}

electron fluid: $\boldsymbol{E}=-\boldsymbol{v}_{\boldsymbol{e}} \times \boldsymbol{B}$. When plasma is disturbed by some external force, electrons react faster to the changes due to their smaller inertia than ions. Thus, electrons thus stay frozen into a magnetic field but the ions lose their magnetic connection due to their larger inertia. The inertia length, $c / \omega_{p}=\sqrt{\frac{c^{2} m \epsilon_{0}}{n e^{2}}}$, is $\sim 40$ times larger for ions than for electrons. If we assume plasma number densities between 5$11 \mathrm{~cm}^{3}$, the ion inertia length varies between $68-100 \mathrm{~km}$.

In the following we will briefly summarize the main results of the non-MHD simulation studies of the KHI before discussing the results of the present study.

Fujimoto and Terasawa (1991) included both the Hall term and electron pressure term in the generalized Ohm's law, linearized and numerically solved these extended MHD equations in parallel $\left(\boldsymbol{V}_{\mathbf{0}} \| \boldsymbol{B}_{\mathbf{0}}\right)$ and perpendicular $\left(\boldsymbol{V}_{\mathbf{0}} \perp \boldsymbol{B}_{\mathbf{0}}\right)$ configurations. Their simulation results indicate that ion inertia effect neither alters the growth rates significantly, nor does it alter the fastest growing wave numbers of the $\mathrm{KH}$ instability compared to the MHD cases. They found, however, that the interior structure of the surface ripples seems to be more complicated than the corresponding results from the MHD analysis indicate, and that the ion inertia effect is most enhanced for the parallel configuration in a low $\beta$ plasma.

Terasawa et al. (1992) observed fast anomalous ion mixing in a hybrid code simulation (particle ions and a massless electron fluid) in perpendicular configuration. Their simulation results show that the mixing occurs within a time scale comparable to the roll-up time of the Kelvin-Helmholtz vortex. They consider this process to be responsible for generating the low-latitude boundary layer.

Fujimoto and Terasawa (1994) further studied this anomalous mixing in a perpendicular configuration assuming a constant magnetic field across the initial shear flow layer. They conclude that the mixing layer formed by the KH instability is independent of the initial thickness of the boundary and that this mechanism could be in part responsible for generating the LLBL. In a companion paper Fujimoto and Terasawa (1995) consider a more realistic configuration where the background magnetic field is inhomogeneous but still perpendicular to the initial shear flow found. They found that including the background inhomogeneity reduces ion mixing.

Huba (1994) studied the effect of the Hall term on the Kelvin-Helmholtz instability in perpendicular configuration. $\mathrm{He}$ found that the maximum velocities perpendicular to the initial shear flow layer are smaller for Hall-MHD than for ideal MHD cases, concluding that the Hall term acts to inhibit the vortex formation, as well as to relax sharp density and magnetic field gradients. He also reported an enhanced diffusion of the magnetic field into the high density, low magnetic field region.

Huba (1996) used a 2-D finite larmor radius (FLR) MHD code to study the nonlinear evolution of the KH instability in a perpendicular, low plasma beta configuration. His simulation results indicate that the FLR effects can either increase or decrease the growth rate of KH instability, depending on the sign of $\boldsymbol{B} \cdot(\nabla \times \boldsymbol{V})$. Similarly, as in the earlier work (Huba, 1994), he suggests that the Kelvin-Helmholtz turbulence may be asymmetric with respect to the dawn and dusk flanks (vorticity changes sign at dawn and dusk), if the boundary layer is sufficiently thin.

Thomas (1995) studied the KH instability with 3-D kinetic simulations using a uniform density embedded in the uniform magnetic field, but let the relative angle of the magnetic field and the velocity vector vary. He found that when $\boldsymbol{V}_{0} \cdot \boldsymbol{B}=0$, the ion mixing property in 2-D and 3-D simulations are almost the same. When $\boldsymbol{V}_{0} \cdot \boldsymbol{B} \neq 0$, the mixing is strongly reduced in 2-D but the 3-D results are not reduced by much.

Most (except Thomas (1995)) of the previous work (Fujimoto and Terasawa, 1991; Terasawa et al., 1992; Thomas and Winske, 1993; Fujimoto and Terasawa, 1994, 1995; Huba, 1994, 1996), that include the ion inertia term or finite larmor radius effect on their simulations, use a perpendicular $\left(\boldsymbol{V}_{\mathbf{0}} \perp \boldsymbol{B}\right)$ configuration or a linearized set of extended MHD equations, or both. In this study we investigate the effects of the Hall term on the evolution and dynamics of the KH instability for different initial configurations. We also complete our 2-D study of plasma transport associated with the KH instability by extending the MHD results to the Hall-MHD regime and consider a realistic, asymmetric plasma and magnetic field configuration, where the magnetic field has a component both perpendicular and parallel to the $\boldsymbol{k}$-vector of the instability. Our main motivation is to understand the ion inertia effect on reconnection inside Kelvin-Helmholtz vortices and whether this effect will alter the net plasma transport quantified with MHD simulations by Nykyri and Otto (2001).

\section{Numerical method}

The results in this study are obtained by solving a full set of resistive two-dimensional Hall-MHD equations:

$$
\begin{aligned}
& \frac{\partial \rho}{\partial t}=-\nabla \cdot(\rho \boldsymbol{v}) \\
& \frac{(\partial \rho \boldsymbol{v})}{\partial t}=-\nabla \cdot\left[\rho \boldsymbol{v} \boldsymbol{v}+\left(\frac{1}{2}\right)\left(p+\boldsymbol{b}^{2}\right) \boldsymbol{I}-\boldsymbol{b} \boldsymbol{b}\right] \\
& \frac{\partial \boldsymbol{b}}{\partial t}=\nabla \times\left(\boldsymbol{v} \times \boldsymbol{b}-\eta \boldsymbol{j}+\frac{1}{n e}(\boldsymbol{j} \times \boldsymbol{b})\right)
\end{aligned}
$$


Table 2. Simulation Runs.

Empty blocks indicate no change from the line above

\begin{tabular}{lrrrrrrrrr}
\hline & $k a$ & $\varphi$ & $B_{1}$ & $B_{2}$ & $n_{1}$ & $n_{2}$ & $\frac{\Delta \rho}{\Delta y_{\rho}}$ & res. & vis. \\
\hline Run 1 & 0.25 & $90^{\circ}$ & 0.15 & 0.1 & 1. & 1. & 0 & 2 & 0.04 \\
Run 2 & 0.25 & $90^{\circ}$ & 0.15 & 0.1 & 1.65 & 0.35 & 0.59 & 2 & 0.04 \\
Run 3 & 0.12 & $3^{\circ}$ & 1.5 & 1.0 & 1.75 & 0.25 & 0.25 & 1 & 0.015 \\
Run 4 & & $5^{\circ}$ & & & & & & & \\
Run 5 & & $10^{\circ}$ & & & & & & & \\
Run 6 & & $15^{\circ}$ & & & & & & & \\
Run 7 & & $25^{\circ}$ & & & & & & & \\
Run 8 & 0.12 & $3^{\circ}$ & 1.5 & 1.0 & 1.65 & 0.35 & 0.59 & 2 & 0.04 \\
Run 9 & & $5^{\circ}$ & & & & & & & \\
Run 10 & & $10^{\circ}$ & & & & & & & \\
Run 11 & $15^{\circ}$ & & & & & & & \\
Run 12 & $25^{\circ}$ & & & & & & & \\
Run 13 & 0.25 & $3^{\circ}$ & 1.5 & 1.0 & 1.65 & 0.35 & 0.59 & 2 & 0.04 \\
Run 14 & & $5^{\circ}$ & & & & & & & \\
Run 15 & $10^{\circ}$ & & & & & & & \\
Run 16 & $15^{\circ}$ & & & & & & & \\
Run 17 & $25^{\circ}$ & & & & & & & \\
\hline
\end{tabular}

$\frac{\partial h}{\partial t}=-\nabla \cdot(h \boldsymbol{v})+\left[\frac{(\gamma-1)}{\gamma}\right] h^{1-\gamma} \eta \boldsymbol{j}^{2}$

$\boldsymbol{j}=\nabla \times \boldsymbol{b}$.

The above partial differential equations are discretized using the finite differences leapfrog scheme (Potter, 1973). The MHD code using this scheme has been developed and discussed in more detail by Otto (1990).

All quantities are normalized to characteristic values for the system, i.e. length scales $l$ to a ion inertia length $L_{0}=c / \omega_{p i}$; density $\rho$ to $\rho_{0}=n_{0} m_{0}$, with the number density $n_{0}$ and the ion mass $m_{0}$; magnetic field $\boldsymbol{b}$ to $B_{0}$; velocity $v$ to the typical Alfvén velocity $v_{A}=B_{0} / \sqrt{4 \pi \rho_{0}}$; pressure to $P_{0}=B_{0}^{2} /(8 \pi)$; current density to $J_{0}=c B_{0} /\left(4 \pi L_{0}\right)$; and time $t$ to a characteristic Alfvén transit time $\tau_{A}=L_{0} / v_{A}$. The values for the normalization of the simulation units are summarized in Table 1.

The initial configuration for the simulation in the boundary coordinates uses a magnetic field of $b_{x 0}(x)=b_{0}(x) \sin \varphi$, $b_{y 0}(x)=0$, and $b_{z 0}(x)=b_{0}(x) \cos \varphi$. The velocity is given by $v_{x 0}(x)=v_{0}(x), v_{y 0}(x)=0$, and $v_{z 0}(x)=0$. Initial density, pressure, velocity, and magnetic field magnitudes are

$$
\begin{aligned}
& \rho_{0}(x)=\frac{1}{2}\left(\rho_{s h}+\rho_{s p}\right)+\frac{1}{2}\left(\rho_{s h}-\rho_{s p}\right) \tanh \left(\frac{y}{L_{0}}\right) \\
& p_{0}(x)=\frac{1}{2}\left(p_{s h}+p_{s p}\right)+\frac{1}{2}\left(p_{s h}-p_{s p}\right) \tanh \left(\frac{y}{L_{0}}\right) \\
& v_{0}(x)=-\frac{1}{2} v_{s h}\left(\tanh \left(\frac{y}{L_{0}}\right)+1\right) \\
& b_{0}(x)=\frac{1}{2}\left(b_{s h}+b_{s p}\right)+\frac{1}{2}\left(b_{s h}-b_{s p}\right) \tanh \left(\frac{y}{L_{0}}\right) .
\end{aligned}
$$

We study the effect of the Hall term in two different system sizes. The smaller system uses a simulation box of 25.2 units in $x$, corresponding to a wavelength of about $0.4 R_{E}$ or $k a=0.25$, where $a$ is the thickness of the velocity shear layer and $k$ is the wave number.

The problem with the inclusion of the Hall term is the increasing phase speed of the Whistler waves for short wavelengths. The Whistler phase speed, $v_{p h}=\omega / k$, is proportional to its $k$ vector and increases linearly with the inverse grid spacing $\frac{1}{\Delta x}$, because better resolution allows for a smaller wavelength of the Whistler mode. The larger phase speed requires better temporal resolution, and we achieved numerical stability by choosing a sufficiently small time step of $\Delta t=0.02$. In addition, a large size of the simulation domain in $y$ of 45.2 (approximately $0.7 R_{E}$ ) is used to minimize the influence of the boundary conditions on the evolution of the $\mathrm{KH}$ waves, for instance, by the reflection of waves (including whistler waves). We use a nonuniform grid in $y$, with maximum resolution of 0.1 in the center of the initial shear flow layer and a uniform grid in $x$ with resolution of 0.12 .

The larger system uses a simulation box of 51.2 units in $x$, corresponding to a wavelength of about $0.8 R_{E}$ or $k a=0.12$. The simulation domain in $y$ is 102.4 (approximately $1.6 R_{E}$ ). The spatial and temporal resolution are the same as that for the smaller system size.

We study the influence of the Hall term with 17 different plasma and magnetic field configurations. The first two simulation sets have a magnetic field only along the $\boldsymbol{k}$-vector of the instability and the remaining 15 simulation sets have a magnetic field in both the $z$-direction and along the $\boldsymbol{k}$-vector. Simulation runs are listed in Table 2 in simulation units, and indices 1, 2 refer to magnetosheath and magnetospheric side, 

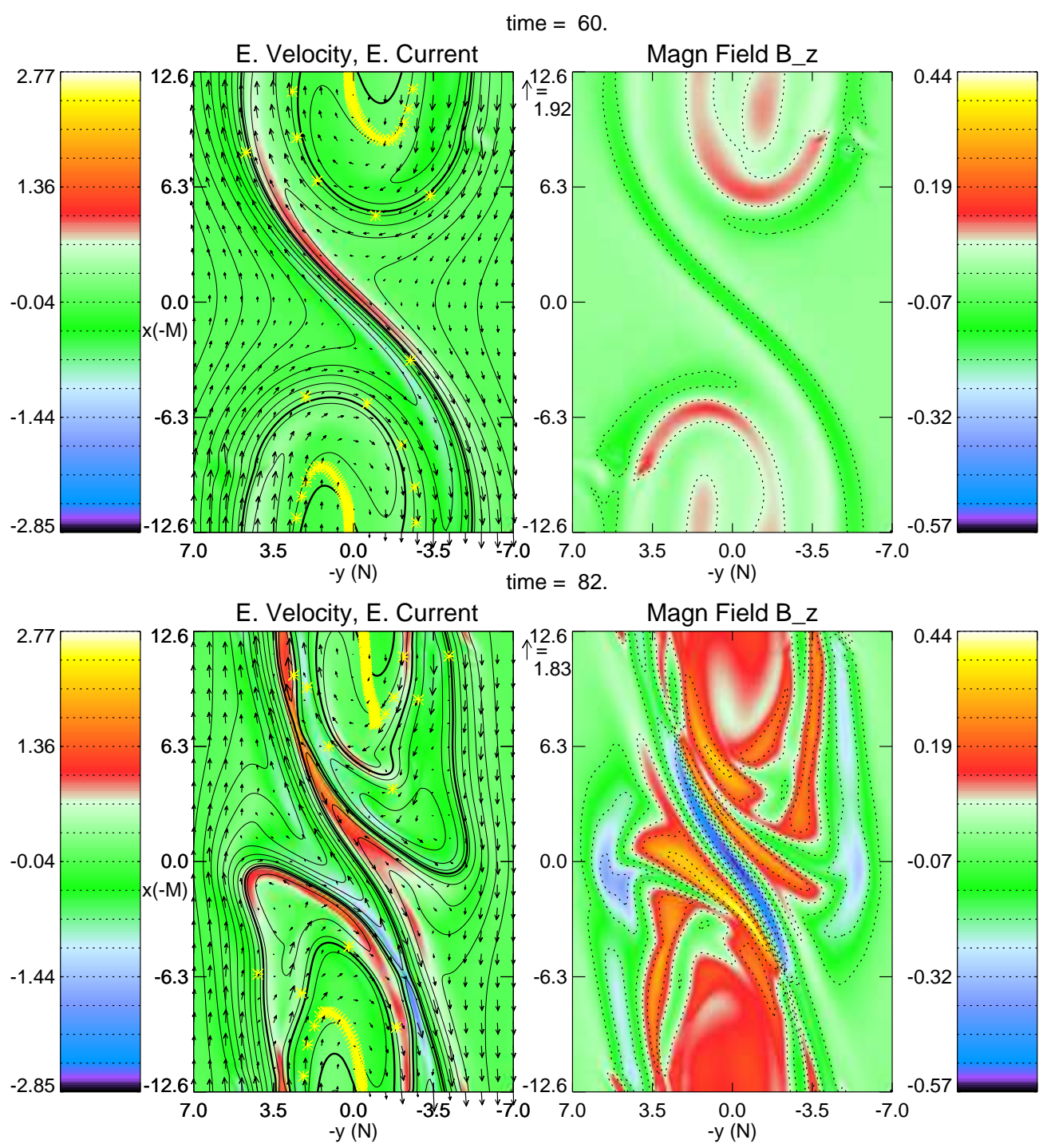

Fig. 1. $\mathrm{KH}$ vortex at $t / \tau_{A} \approx 60$ and at $t / \tau_{A} \approx 82$. Lines are magnetic field lines projected to $x, y$-plane and arrows are electron velocity vectors. Color code represents electron current (left) and $z$ component of the magnetic field (right).

respectively. Empty blocks indicate no change in the parameters from the line above.

Simulation runs 1-7 have the same parameters as the MHD simulations in Nykyri and Otto (2001), except for a larger simulation domain in $x$-direction. In addition to the simulation runs listed in Table 2, we have simulated cases 8-12 with the MHD code (with exactly the same parameters) for comparison. Runs 13-17 are shorter wavelength runs that are compared with longer wavelength cases $8-12$. We use the following two resistivity models in these simulations:

$$
\begin{array}{lr}
\eta(j)=\kappa \sqrt{\left(j^{2}-J_{c}^{2}\right)} S\left(j^{2}-J_{c}^{2}\right) & (\text { model } 1) \\
\eta(j)=\kappa\left(j^{2}-J_{c}{ }^{2}\right)^{2} S\left(j^{2}-J_{c}{ }^{2}\right) & (\text { model }) .
\end{array}
$$

Here, $\kappa=0.005$ and $J_{c}=1.1 J_{0}$ for model 1 which corresponds to model 1a) in MHD simulations of Nykyri and Otto (2001).
Model 2 uses $\kappa=0.001$ and $J_{c}=2.5 J_{0} . S$ is a step function equal to unity for arguments greater than 0 and equal to 0 otherwise.

\section{Demonstration of the Hall effect (simulation results without background $B_{z}$ )}

\subsection{Magnetic field asymmetry (Run 1)}

Figures 1 and 2 show the evolution of the Kelvin-Helmholtz wave for Run 1 at $t / \tau_{A} \approx 60$ and at $t / \tau_{A} 82$. In Fig. 1 lines are magnetic field lines projected to $x, y$-plane and arrows are electron velocity vectors. The electron current (left) and the $z$ component of the magnetic field (right) are color coded. Yellow asterisks are fluid elements that are located initially at the boundary $y=0$. At $t / \tau_{A} \approx 60$ a vortex in electron velocity field is clearly visible, with narrow electron current layers 

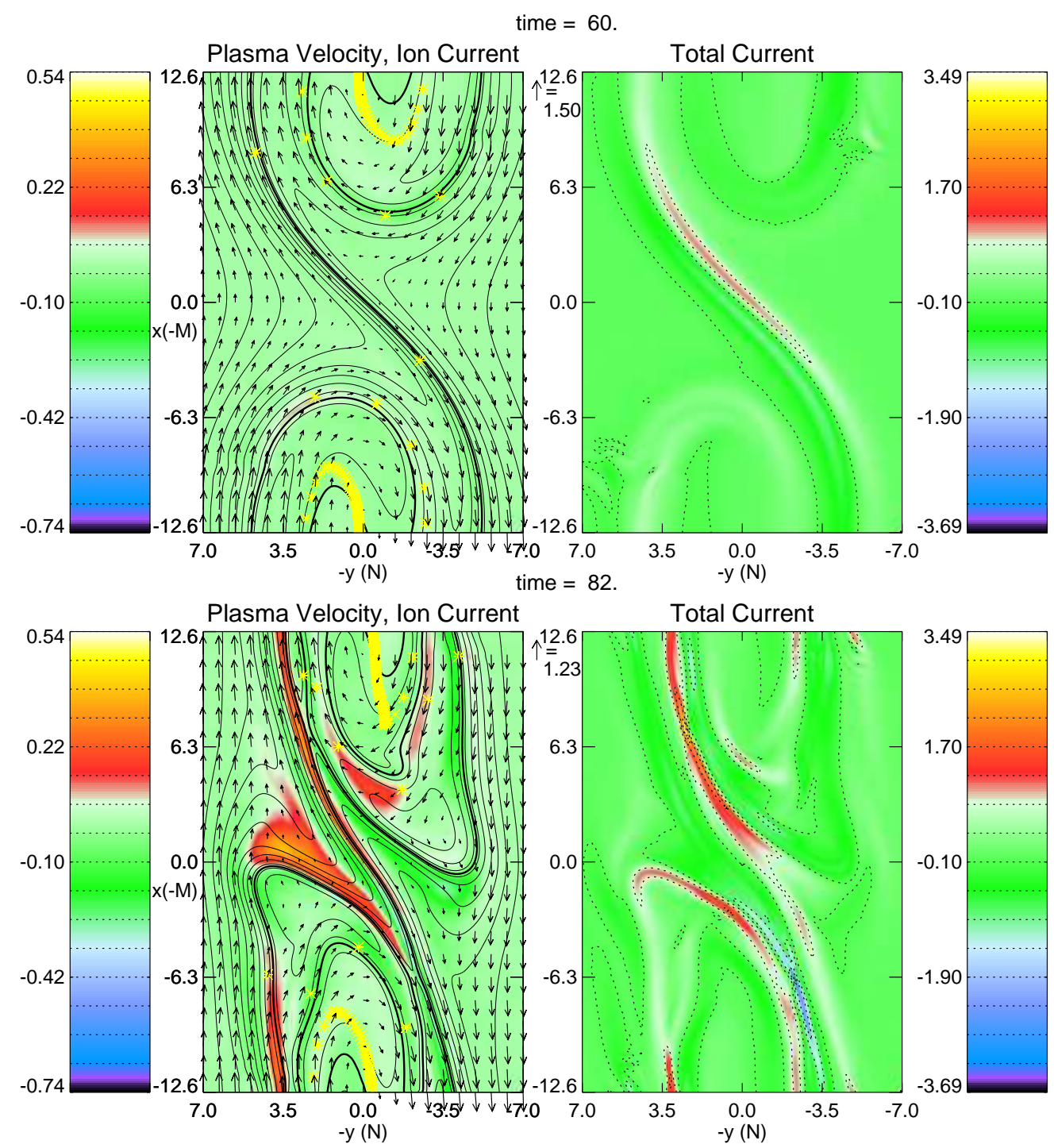

Fig. 2. $\mathrm{KH}$ vortex at $t / \tau_{A} \approx 60$ and at $t / \tau_{A} \approx 82$. Lines are magnetic field lines projected to $x, y$-plane and arrows are plasma velocity vectors. Color code represents ion current (left) and total current (right).

of opposite polarity forming in the center of the simulation box. The Hall effect also starts generating an out-of-theplane magnetic field component. At $t / \tau_{A} \approx 82$ a highly nonlinear vortex has formed with very strong electron current layers. Ongoing reconnection is observable in the positive electron current layer at $x,-y=[7.3,2.8]$ and at negative electron current layer at $x,-y=[-6.3,-2.7]$. Reconnection is not symmetric because the initial configuration had a magnetic field asymmetry. If the magnetic field is exactly the same on both sides of the initial shear flow layer, magnetic reconnection would happen at the same rate in both negative and positive current layers forming a symmetric structure. Strong out-of-the-plane magnetic field components are generated such that the strongest, negative $B_{z}$ layer is formed in the electron outflow region from the reconnection site at $x,-y=[7.3,2.8]$ (towards the center of the simulation box). The out-of-plane magnetic field has the same polarity as in the Hall-MHD reconnection challenge (Otto, 2001) (when reconnection operates in a positive electron current layer, a positive $B_{z}$ is formed in the regions where outflow is in the $+x$-direction, and a negative $B_{z}$ in the regions of the $-x$-direction. In the negative current layer this behaviour is reversed) and is consistent also in magnitude.

In Fig. 2 arrows are plasma velocity vectors, the color code represents ion current (left) and total current (right). At $t / \tau_{A} \approx 60$ a vortex is clearly visible but there is no ion current. The total current is dominated by the electrons. At $t / \tau_{A} \approx 82$ the ion current increases in two regions where there is no electron current and where the plasma velocity is very slow. This is probably due to the Hall electric field generated by the poloidal electron flow across the poloidal magnetic field. The total current which is mostly carried by electrons has a maximum in reconnection regions at $x,-y=[6.3,2.0]$ and at $x,-y=[-6.3,-2.7]$. 

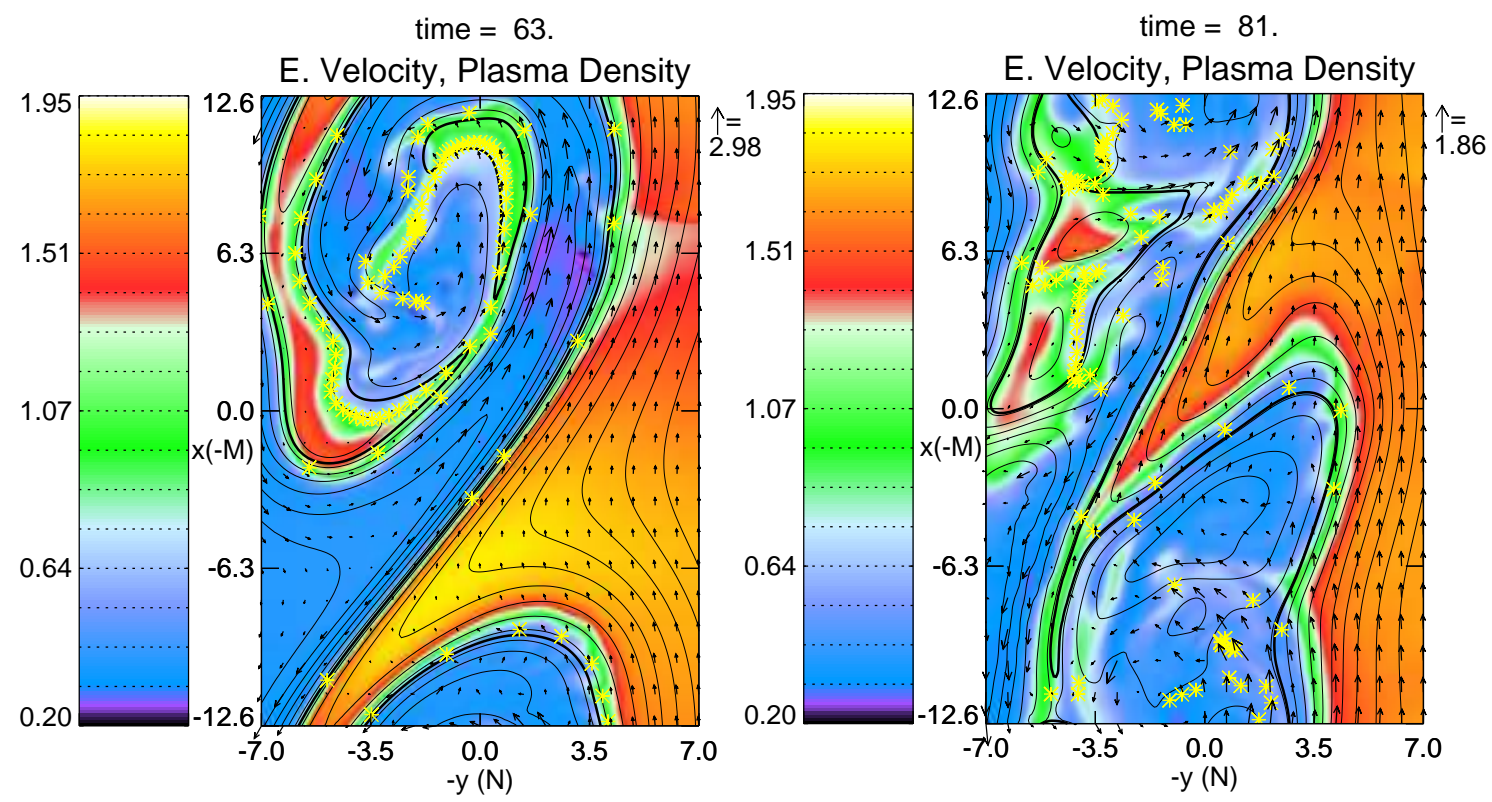

Fig. 3. KH vortex at $t / \tau_{A} \approx 63$ and at $t / \tau_{A} \approx 81$. Lines are magnetic field lines projected to $x, y$-plane and arrows are electron velocity vectors. Color code represents plasma density.

\subsection{Magnetic field and density asymmetry (Run 2)}

To illustrate the effect of an added initial density asymmetry, Fig. 3 depicts a KH vortex at $t / \tau_{A} \approx 63$ and at $t / \tau_{A} \approx 81$ for the Run 2 parameters. Lines are magnetic field lines projected to $x, y$-plane and arrows are electron velocity vectors. The color code represents plasma density. The KelvinHelmholtz instability twists the magnetic field in the $x, y$ plane which leads to anti-parallel magnetic field components. A high density magnetic island becomes reconnected at $t / \tau_{A} \approx 63$, and a second island is detached at $t / \tau_{A} \approx 81$. Compared to Run 1 , the reconnection occurs $20 \tau_{A}$ earlier for Run 2. The inertia of the vortex motion in the high density spine is larger than in the lower density layer, causing the reconnection to occur earlier for Run 2. For the cases considered in this study (Runs 2-17) the higher density spine is always on the same side as the higher current density layer, because $B_{1}$ and $n_{1}$ are larger than $B_{2}$ and $n_{2}$ (Table 2). An interesting future study would be to investigate a reconnection balance between high-density, low-current-spine and the low-density, high-current-spine.

For the same run Fig. 4 shows magnetic field lines projected to the $x, y$-plane and arrows are electron velocity vectors. The electron current (left) and the $z$ component of the magnetic field (right) at $t / \tau_{A} \approx 63$ (top) and at $t / \tau_{A} \approx 81$ (bottom)are color coded. At $t / \tau_{A} \approx 63$ reconnection occurs at $x,-y=[3,0]$ in a region of negative electron current. A positive, elongated electron current layer is located adjacent to a strong magnetic field layer. A weak, negative $B_{z}$ layer starts forming in the elongated regions of enhanced magnetic field. At $t / \tau_{A} \approx 81$ a second magnetic island is detached at $x,-y=[-11,-5]$ and the negative $B_{z}$ layers are intensified.

In Fig. 5 arrows are plasma velocity vectors and the color code represents ion current (left) and total current (right) at $t / \tau_{A} \approx 63$ and at $t / \tau_{A} \approx 81$. At $t / \tau_{A} \approx 63$ (top) the first magnetic island is detached in the region where total current (top right) is negative. At $t / \tau_{A} \approx 81$ (bottom) a second magnetic island is detached at $x,-y=[-11,-5]$ in the negative current layer. The positive current has increased from $t / \tau_{A} \approx 63$, but it is not distributed only along the elongated layer like at $t / \tau_{A} \approx 63$. Compared to the previous run the magnitude of the ion current has remained closely the same but it is now occupying a larger area. These differences are due to the change in initial condition: the density asymmetry changes the dynamics of $\mathrm{KH}$ instability.

\section{Results of the plasma transport}

In Nykyri and Otto (2001) the plasma transport associated with the Kelvin-Helmholtz instability was estimated using an MHD approximation. Here, we study the influence of the Hall effect on the plasma transport and compare the results with the MHD cases.

The mass transport is calculated by integrating the plasma density over magnetic islands (flux ropes) captured from the magnetosheath, i.e. islands of magnetosheath material which are entirely embedded in the magnetosphere. The method of defining the initial boundary is similar to the one discussed in Nykyri and Otto (2001) but here the magnetic islands are defined by electron fluid elements. During the simulation, the location of these elements is determined by integrating the electron fluid velocity. Ideally, the electron fluid elements stay on the same field line if the magnetic field is frozen into the electron fluid flow. However, reconnection and numerical dissipation can break this condition. 

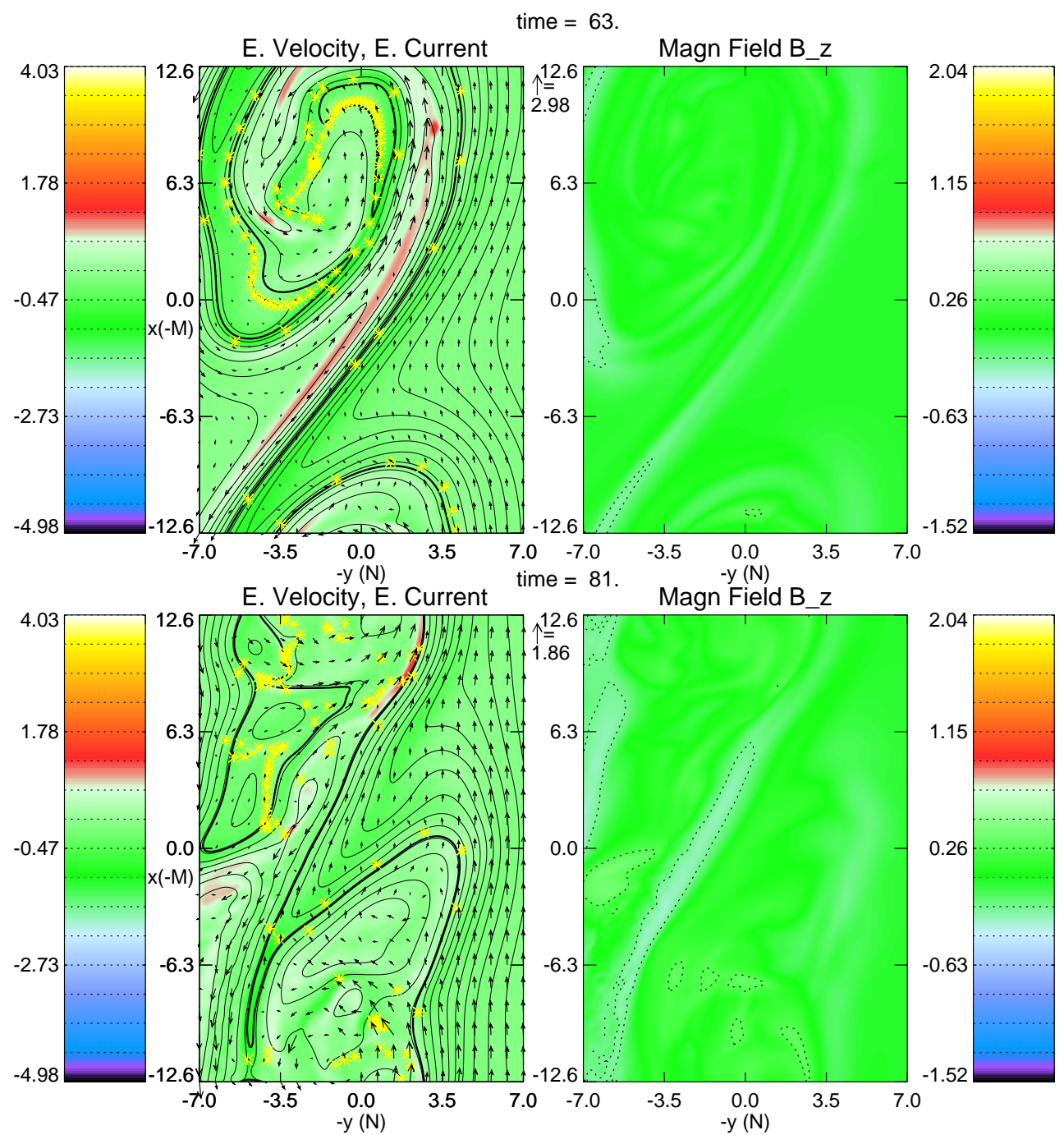

Fig. 4. $\mathrm{KH}$ vortex at $t / \tau_{A} \approx 63$ and at $t / \tau_{A} \approx 81$. Lines are magnetic field lines projected to $x, y$-plane and arrows are electron velocity vectors. Color code represents electron current (left) and $z$ component of the magnetic field (right).

In two dimensions Ohm's law (including the Hall term) can be written as

$\frac{\partial A_{z}(t)}{\partial t}+\left(\mathbf{V}_{\mathbf{e}} \cdot \nabla\right) A_{z}(t)=-\eta J_{z}$

where $A_{z}$ is the $z$-component of the magnetic vector potential, and changes along the path of a fluid element are only due to the resistive term on the right side of (1), if the initial gauge of $A_{z}$ is maintained. If this is the case, a fixed value of $A_{z}$ determines the magnetic boundary. Since $A_{z}$ is determined by integrating the magnetic field, we use the electron fluid elements to determine the proper gauge and to test the frozen-in condition.

The mass entry from the magnetosheath into the magnetosphere is calculated by integrating the density over the area of the detached magnetic islands. An averaged mass entry rate is determined by dividing the mass in the islands through a typical time (i.e. the time it takes to form a nonlinear wave).
The results in Figs. 7, 8, and 9 are expressed in terms of an average entry velocity

$v_{\text {entry }}=\frac{\Delta M}{\Delta t} \frac{1}{\rho_{s h} L_{x}}$,

where $M$ is the mass of the magnetic island, $\Delta t$ is the simulation time, $L_{x}$ is the wavelength and $\rho_{s h}$ is the magnetosheath density.

4.1 Demonstration of plasma transport and growth rates (Runs 3-7)

Figure 6 shows the evolution of the Kelvin-Helmholtz wave for Run 5 at $t / \tau_{A} \approx 62$ and at $t / \tau_{A} \approx 107$. Lines are magnetic field lines projected to $x, y$-plane and arrows are electron velocity vectors. Color represents plasma density (upper panel) and the $z$-component of the magnetic field (lower panel). The first magnetic island is detached at $t / \tau_{A} \approx 62$, and there is a depletion of the $B_{z}$-component surrounding the reconnected 

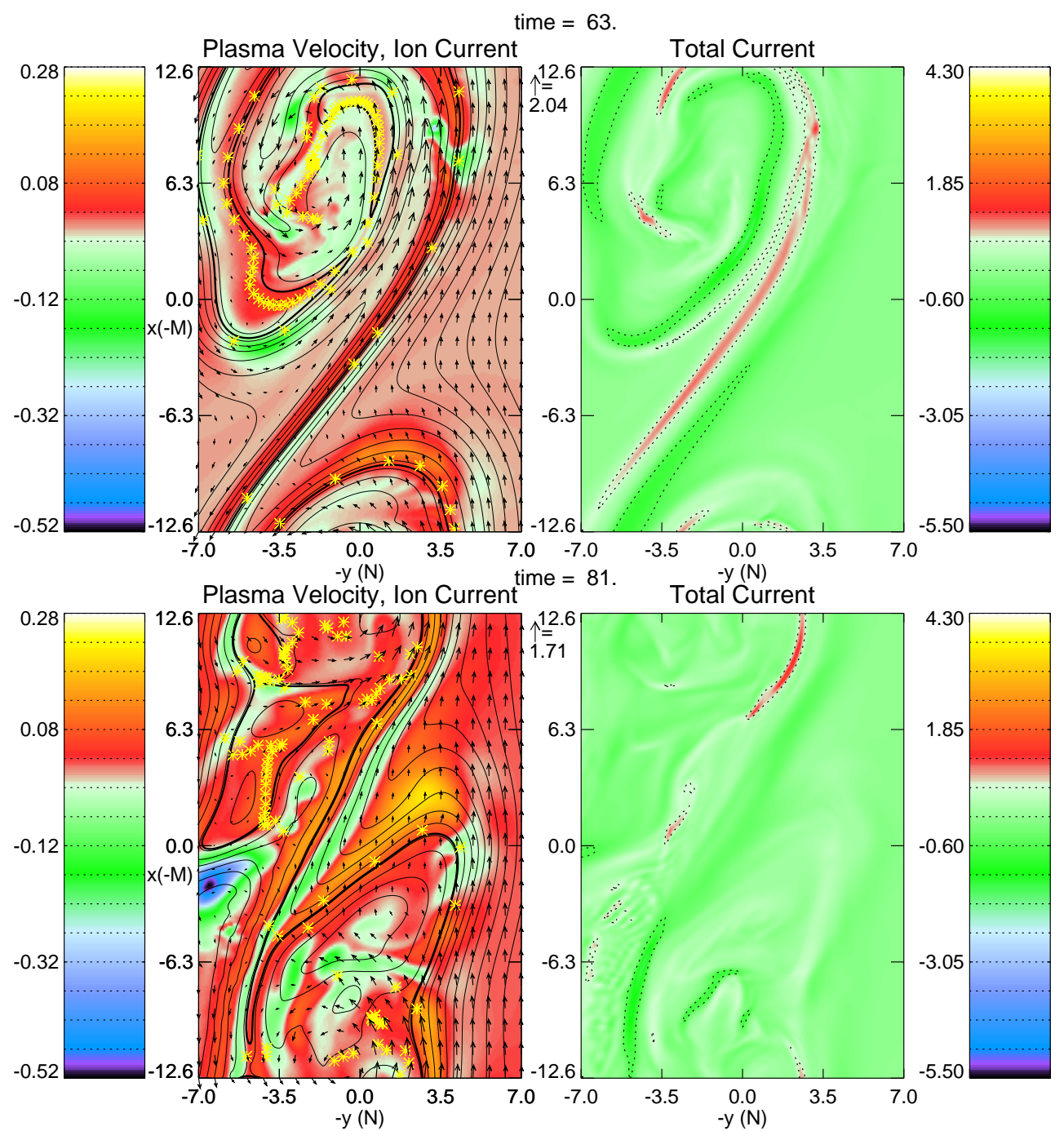

Fig. 5. $\mathrm{KH}$ vortex at $t / \tau_{A} \approx 63$ and at $t / \tau_{A} \approx 81$. Lines are magnetic field lines projected to $x, y$-plane and arrows are plasma velocity vectors. Color code represents ion current (left) and total current (right).

island. Formation of this first island corresponds to the first peak in mass transport velocity in Fig. $7 \mathrm{a}$. At $t / \tau_{A} \approx 107$ the main magnetic island is detached by reconnection from the magnetosheath corresponding to the maximum mass diffusion velocity of $1.2 \mathrm{~km} / \mathrm{s}$ in Fig. 7a.

Figure $7 \mathrm{~b}$ illustrates the effect of the magnetic field along the $\boldsymbol{k}$-vector of the KH instability for Runs 37. Plasma transport velocities are reduced for increasing $\varphi\left(B_{x}=B_{0} \sin (\varphi)\right)$, due to the stabilization of the KH instability by the magnetic field. Errors in the average velocity can be caused by the choice of $\Delta t$ and inaccuracies of the island boundaries.

Figure $7 \mathrm{c}$ depicts the growth of the Kelvin-Helmholtz instability as a function of $t / \tau_{A}$ for $\varphi=3^{\circ}, 5^{\circ}, 10^{\circ}, 15^{\circ}$ and $25^{\circ}$. Naturally, the largest growth rate, $q=0.1$, is obtained for $\varphi=3^{\circ}$ and the smallest growth rate, $q=0.09$, is obtained for $\varphi=25^{\circ}$. So the maximum linear growth rate is only about $10 \%$ larger than the minimum growth rate, but the corresponding maximum mass transport velocities for and $25^{\circ}$ dif- fer by about $88 \%$. Growth rates in this study are obtained by plotting (in log-scale) the maximum values of perpendicular velocity components during the whole simulation as a function of time and calculating the slope of the growth graph (slope of the graph $\ln \left(v_{y}\right)$ vs $t$ in Figs. 7, 8, 9).

\subsection{Comparison of plasma transport and growth rates be- tween MHD and Hall-MHD simulations (Runs 8-12)}

Figure 8a represents mass transport velocities as a function of $\varphi$ for simulation runs 8-12. For comparison we also simulated these cases with the MHD code. Both plasma approximations give the same results within the limits of the error bars. The maximum plasma transport velocity, $V_{\text {diff }}=1.8 \mathrm{~km} / \mathrm{s}$ is obtained for $\varphi=3^{\circ}$. The plasma transport is reduced for large $\varphi$ due to the stabilization by the magnetic field.

Figures $8 b$ and $c$ show growth of the Kelvin-Helmholtz instability for the Hall-MHD and MHD approximations as a 

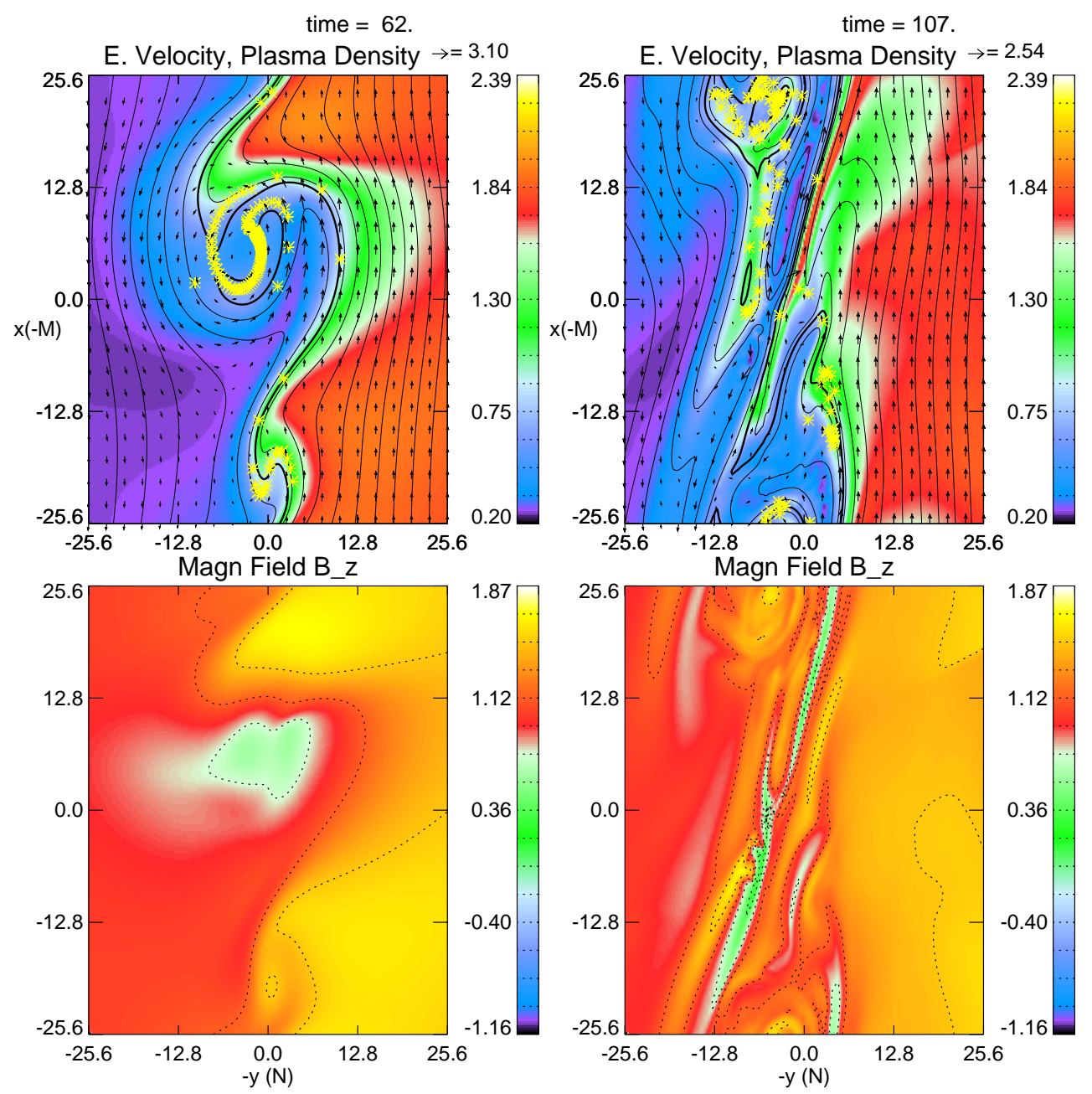

Fig. 6. KH vortex at $t / \tau_{A} \approx 62$ and at $t / \tau_{A} \approx 107$ for Run 5 . Lines are magnetic field lines projected to $x, y$-plane and arrows are electron velocity vectors. Color code represents plasma density (upper panel) and $z$-component of the magnetic field (lower panel).

function of $t / \tau_{A}$. The largest growth rate, $q=0.1$, is obtained for the Hall-MHD case with $\varphi=3^{\circ}$ and the smallest growth rate, $q=0.07$, is obtained for the MHD case with $\varphi=25^{\circ}$.

For the Hall-MHD approximation the maximum linear growth rate is $24 \%$ larger than the minimum growth rate. For MHD simulations the maximum growth rate is only $13 \%$ larger than the minimum growth rate. The Hall-MHD growth rates for $\varphi=3^{\circ}, 5^{\circ}, 10^{\circ}, 15^{\circ}$, and $25^{\circ}$ are $21 \%, 17 \%, 13 \%$, $13 \%$ and $7 \%$ larger than the corresponding MHD growth rates, respectively. The growth rates are larger for all the Hall-MHD cases compared to the corresponding MHD cases because in the Hall-MHD the magnetic field is frozen into the electrons and thus, it doesn't constrain the ion motion. This difference between MHD and Hall-MHD-KH growth becomes smaller for larger magnetic field values along the $\boldsymbol{k}$ vector of the KH mode. This appears counterintuitive at first glance because the smaller magnetization of ions due to the Hall physics should be more important for larger magnetic fields along the $\boldsymbol{k}$-vector. However, the Hall effect also generates a perpendicular (out of the plane) magnetic field which is not present in MHD. The energy for this magnetic field gen- eration must come from the kinetic flow energy such that this effect should act to reduce the growth rate for Hall dynamics, in particular for larger magnetic field values along the $\boldsymbol{k}$ vector. Thus, the combined effect of the demagnetized ions and of the perpendicular magnetic field generation may account for the smaller difference in the Hall MHD and MHD growth rates for larger magnetic field along the $\boldsymbol{k}$-vector.

Except for the case with $\varphi=3^{\circ}$, the Hall-MHD growth rates are slightly larger for simulations Runs 3-7 than for cases $8-12$. This is probably due to the lower viscosity and smaller density gradient used in Runs 3-7. Note that the viscosity is employed to arrest nonphysical grid oscillations which can occur because the leapfrog method lacks numerical diffusion. Here, it is used as a velocity diffusion term with a physical coefficient which scales as $\alpha \Delta^{3}=$ where $\Delta$ is the local grid spacing and $\alpha$ is the coefficient listed in Table 2 . For a unit distance the corresponding velocity diffusion time is approximately $10^{3}$ Alfvén times in the region with the best grid resolution, i.e. much longer than the time scales considered in our simulations. Thus, corresponding effects should be small in general, however, a residual effect on growth 

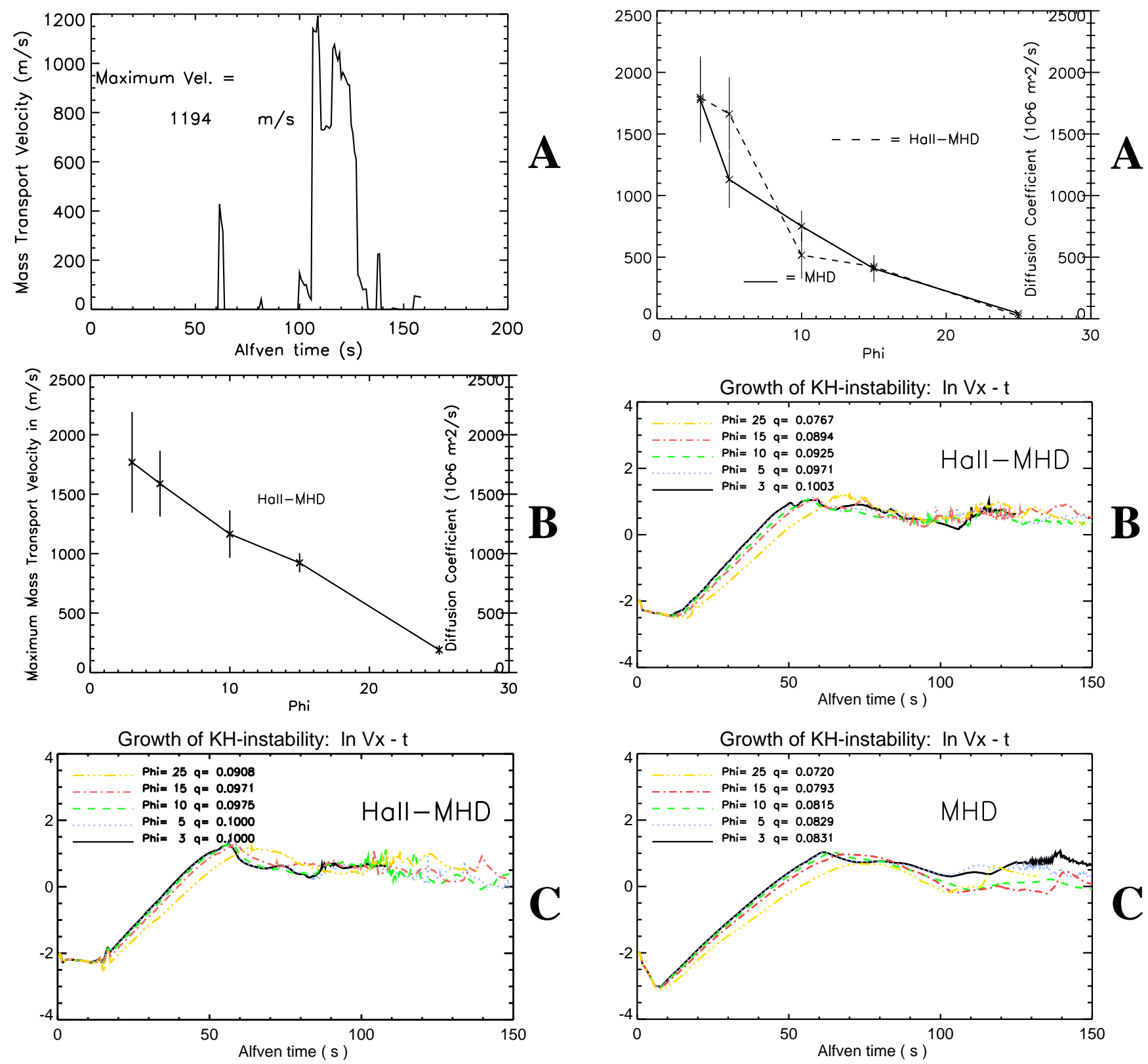

Fig. 7. (A) Mass transport velocity as a function of time for $\varphi=10^{\circ}$. (B) Mass transport velocity as a function of $\varphi$. (C) Growth of $\mathrm{KH}$ instability for $\varphi=3^{\circ}, 5^{\circ}, 10^{\circ}, 15^{\circ}$ and $25^{\circ}$.

rates cannot be excluded as expressed in the slightly different growth rates comparing Runs 3-7 and 8-12.

The Hall-MHD mass transport velocities agree for small $\varphi$, between cases 3-4 and 8-9, but are smaller for cases 10-12 than for cases 5-7. In Nykyri and Otto (2001) it was shown that reconnection inside Kelvin-Helmholtz vortices is independent of the resistivity model since the reconnection is strongly driven by the vortex motion. We assume that the discrepancy between plasma transport velocities for large $\varphi$ is due to a slightly increased stabilization of the $\mathrm{KH}$ mode both by larger viscosity in Runs 8-12 and by the larger magnetic field.

Fig. 8. (A) Mass transport velocity as a function of $\varphi$ for MHD and Hall-MHD. (B) Growth of KH instability for $\varphi=3^{\circ}, 5^{\circ}, 10^{\circ}, 15^{\circ}$ and $25^{\circ}$ for Hall-MHD approximation. (C) Growth of KH instability for MHD approximation.

4.3 Demonstration of the wavelength effect on the plasma transport (Runs 13-17)

Figure 9a represents mass transport velocities for the small wavelength $(k a=0.25)$ cases 13-17. These results agree (within error bars) with mass transport velocities obtained for large wavelength cases $8-12(k a=0.12)$. Figure $9 \mathrm{~b}$ shows KH growth for $\varphi=3^{\circ}, 5^{\circ}, 10^{\circ}, 15^{\circ}$, and $25^{\circ}$. The short wavelength growth rates are about $40 \%$ larger than the large wavelength growth rates. The maximum linear growth rate is $25 \%$ larger than the minimum growth rate for $k a=0.25$. At 
$t / \tau_{A} \approx 60$ this fast growing, small wavelength mode is clearly observable in Fig. 6. It grows faster into a nonlinear stage and decays before reconnection occurs at the vortex of the larger wavelength.

Huba (1994) obtained growth rates of $q \approx 0.04 V_{0} / a$ for both MHD and Hall-MHD approximations for $k a=0.26$. For the values of $V_{0}=315 \mathrm{~km} / \mathrm{s}$ and $a=100 \mathrm{~km}$ used in the simulation study, the growth rate obtained by Huba (1994) becomes $q \sim 0.126$, which is comparable to the values presented in Fig. 9b. Fujimoto and Terasawa (1991) report little affect of Hall dynamics on the linear growth rate of the KelvinHelmholtz instability for $k a<0.4$.

\section{Small-scale structures}

Huba (1994) reported the Hall effect to cause small-level turbulence $(k a>1)$ that inhibits vortex formation. We also found small-level, ion inertia scale vorticity in our simulations. The ion inertia scale vorticity is clearly observable as small ripples in plasma density. We found the most clearly structured, small-scale vortices we found for $\varphi=5^{\circ}$ (Run 4). Figure 10a represents electron velocity (arrows) and plasma density (color code) at $t / \tau_{A} \approx 93$, and Fig. 10b shows the $B_{z}$ component of the magnetic field. Magnetic field lines are not plotted on these figures, in order to distinguish the smallscale structure better. One can see two vortices in Fig. 10a, one corresponding to a $k a=0.12$ and the other to $k a=0.25$. Figure 10c represents a magnified region of Fig. 10a, revealing a chain of small-scale vortices with a wavelength of approximately 1 to $1.5 \mathrm{c} / \omega_{p i}$. Ion inertia scale ripples appeared first in the smaller wavelength $(k a>0.25) \mathrm{KH}$ vortex at $t / \tau_{A} \approx 90$. This structure existed for $9 \tau_{A}$ before the vortex decayed. Small scale vortices are observed for all cases $3-8$, but the longest lasting structures are obtained for small angles $\varphi=3^{\circ}, 5^{\circ}$. In these cases the ion inertia scale perturbations "attack" the high density, small wavelength $(k a=0.25)$, vortex resulting in vortex decay. The dynamical evolution of the larger vortex is not altered by these ion inertial length dynamics. For larger angles $\left(\varphi=10^{\circ}\right.$ and $\left.15^{\circ}\right)$ the ion dynamics doesn't cause a decay of the smaller, high density vortex but works mostly in the "edges" of the higher density region, mixing "intermediate" density plasma with the lowest density plasma. For $\varphi=25^{\circ}$, the small-scale perturbations were strongly reduced. We also studied the effect of zero magnetic field in the simulation plane using a perpendicular configuration $(\boldsymbol{k} \perp \boldsymbol{B})$, to see whether ion inertia scale perturbations are further enhanced from cases with $\varphi=3^{\circ}$ and $5^{\circ}$. For $\varphi=0^{\circ}$, the growth rate of $\mathrm{KH}$ is larger and the overall dynamics is more turbulent due to the lack of magnetic field stabilization, so the system was already in the saturation stage when the small-scale turbulence started affecting a narrow high density layer. It's also worth mentioning that, while Huba (1994) observed small-scale turbulence to inhibit vortex formation, in our simulation the main vortices were already fully developed before the ion inertia scale turbulence started decaying the smaller vortex.
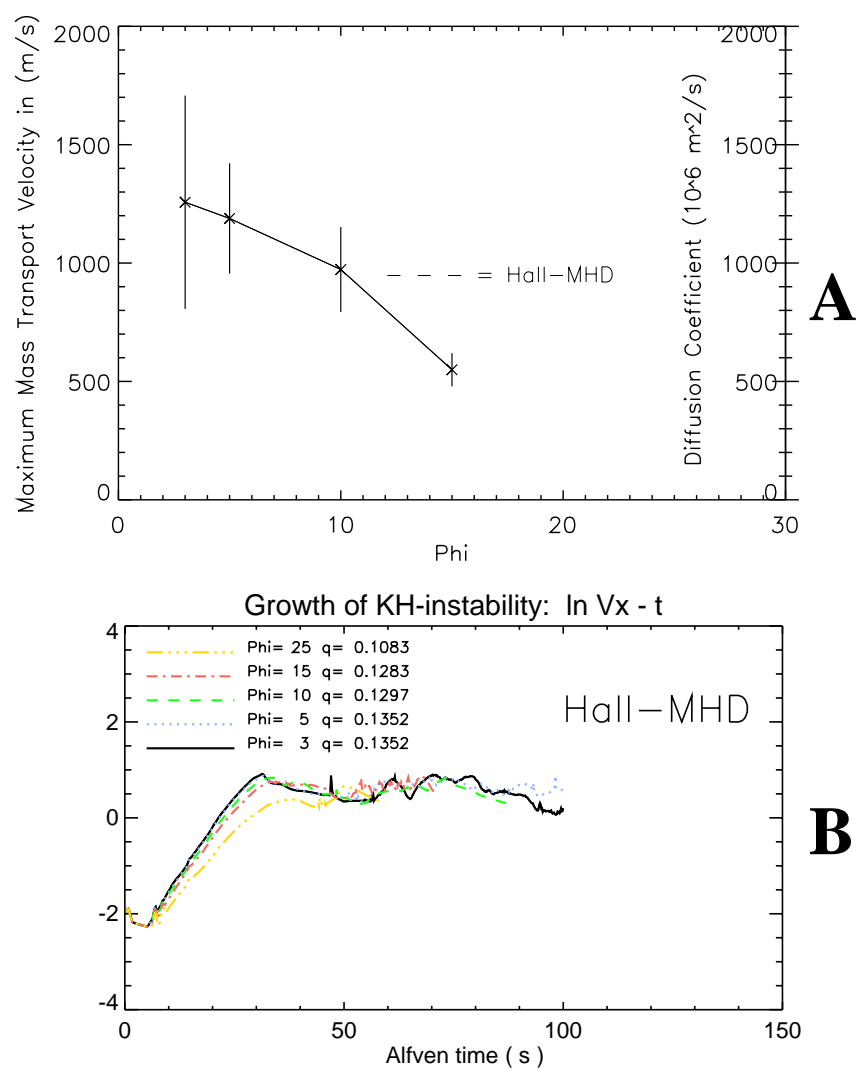

Fig. 9. (A) Mass transport velocity as a function of $\varphi$ for short wavelength ( $k a=0.25, \lambda=0.4 R_{E}$ ) Hall-MHD. (B) Growth rates of KH instability for $\varphi=3^{\circ}, 5^{\circ}, 10^{\circ}, 15^{\circ}$ for Hall-MHD approximation.

Figure 11 shows magnification of Fig. 10a for different plasma and field properties. Figure 11a represents electron velocity (arrows) and plasma density (color code) on the left, and the $z$-component of the magnetic field on the right. Figure $11 \mathrm{~b}$ depicts plasma velocity (arrows) and ion current (color code) on the left, and total current on the right. Magnetic field (arrows) and electron current (color code) are plotted on the left of Fig. 11c and $z$-component of the velocity is plotted on the right. A comparison between Figs. 11a and $11 \mathrm{~b}$ indicates that regions of high plasma density correspond to regions of negative ion current. Similarly, the positive ion current regions correspond to the regions of low plasma density. Positive ion current regions also correlate with regions of positive plasma velocity. The magnetic field reverses sign across the positive electron current layer. Since the magnetic field is frozen into the electron fluid, a depletion in $B_{z}$ is formed in the regions of strong positive electron current (electrons traveling into the $-z$-direction). In the regions of negative ion current, there is a large positive electron current, so in these regions the electrons and protons are traveling into same direction, reducing the total current in these regions. 


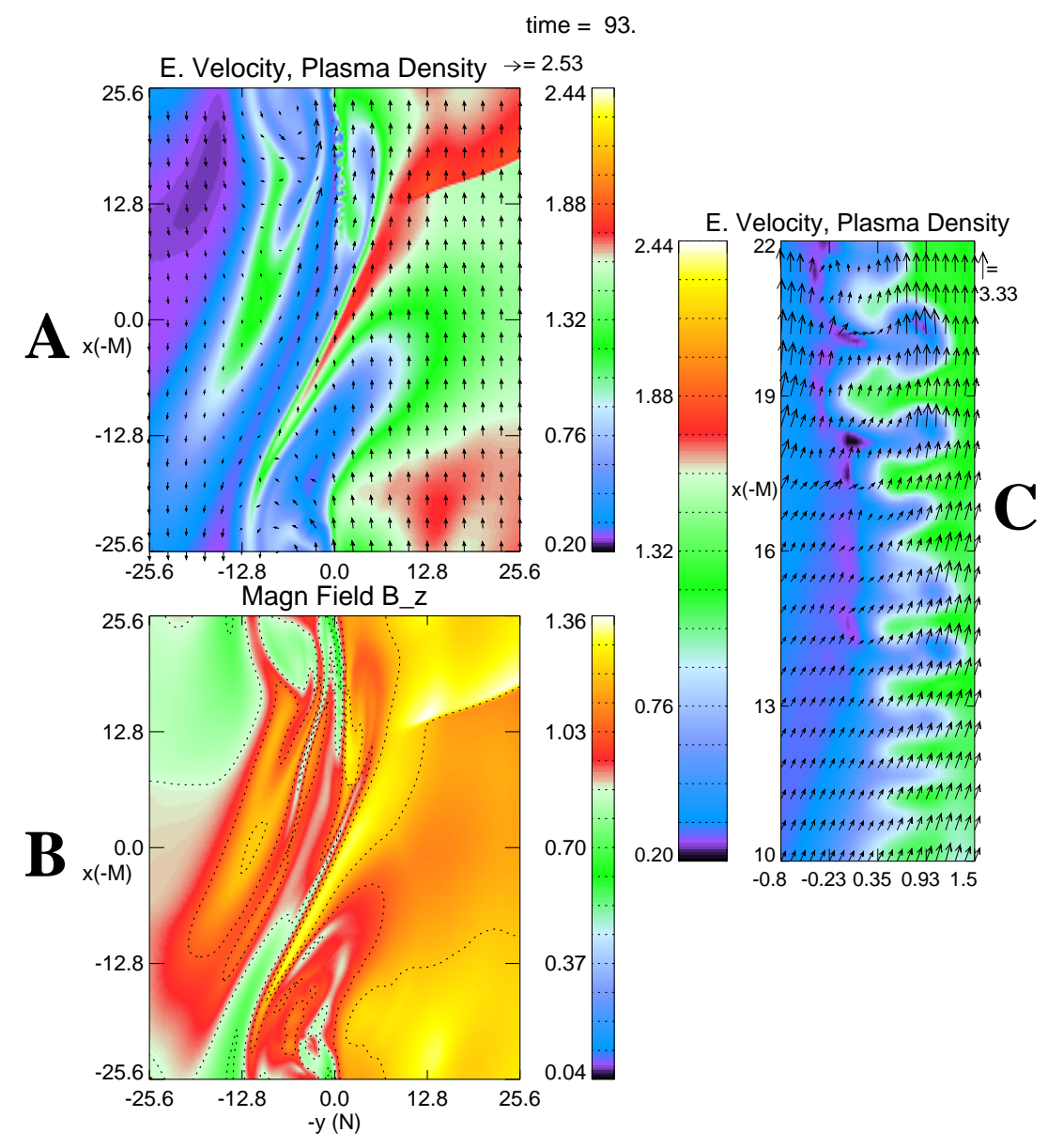

Fig. 10. $\mathrm{KH}$ vortex for $\varphi=5$ at $t / \tau_{A} \approx 93$. (A) shows electron velocity (arrows) and plasma density (color code). (B) represents $z$-component of the magnetic field. (C) is a magnified domain of Fig. A.

\section{Discussion and Conclusions}

In this study it has been demonstrated that the net plasma transport due to reconnection inside Kelvin-Helmholtz vortices is unaltered in a Hall-MHD approximation for the considered cases. For small $\varphi$ cases (corresponding to strongly northward interplanetary magnetic field) we estimate the mass diffusion velocities to be about $1.5 \mathrm{~km} / \mathrm{s}$. If we assume a solar wind density of $11 \mathrm{~cm}^{3}$ and a plasma sheet density of $1 \mathrm{~cm}^{3}$, it would take about $2 \mathrm{~h}$ to replace all the mass in the plasma sheet with this process. This is in excellent agreement with observations by Borovsky et al. (1998) and Fujimoto et al. (1998), which indicate that during periods of strongly northward IMF the cold dense plasma sheet is formed in a time scale of about two hours.

For small $\varphi$, Hall-MHD growth rates are about 20\% larger than the MHD growth rates, and for cases with large magnetic field along the $\boldsymbol{k}$-vector of the instability (large $\varphi$ ), the Hall-MHD growth rates are about 7\% larger. The growth rates are larger for all the Hall-MHD cases compared to the corresponding MHD cases, because in the Hall-MHD the magnetic field is frozen into the electrons and thus, it doesn't stabilize the ion motion on sufficiently small-scales. Fig- ure 12 shows a comparison between linear MHD theory and our simulation results. Figure 12a indicates that the growth rates predicted from the linear, incompressible $(\nabla \cdot v=0)$ theory are about $50 \%$ larger than from the simulations for longer wavelength $(k a>0.12)$ cases. This difference is even larger $(100 \%)$ for shorter wavelength cases $(k a>0.25)$ in Fig. $12 b$. Theory assumes that the velocity shear layer thickness $a$, is infinitesimal, that plasma is incompressible, and that the world is linear. When the wavelength approaches the shear flow layer thickness, the growth of the wave is not possible in reality (and simulations), but for linear theory there is not such a limit.

Huba (1994) concluded that the Hall term acts to inhibit vortex formation, implying that the growth rate for the Kelvin-Helmholtz mode is reduced by ion inertia effects. However, results in this study indicate that Hall-MHD growth rates are about 20\% larger than the corresponding MHD growth rates. Huba (1994) comes to his conclusion by evaluating only the maximum value of the perpendicular velocity component across the initial shear flow layer such that the one maximum value of the whole simulation represents the growth. In MHD approximation Huba (1994) reported the maximum $v_{y}$ to be $40 \mathrm{~km} / \mathrm{s}$, and in Hall-MHD $20 \mathrm{~km} / \mathrm{s}$ 

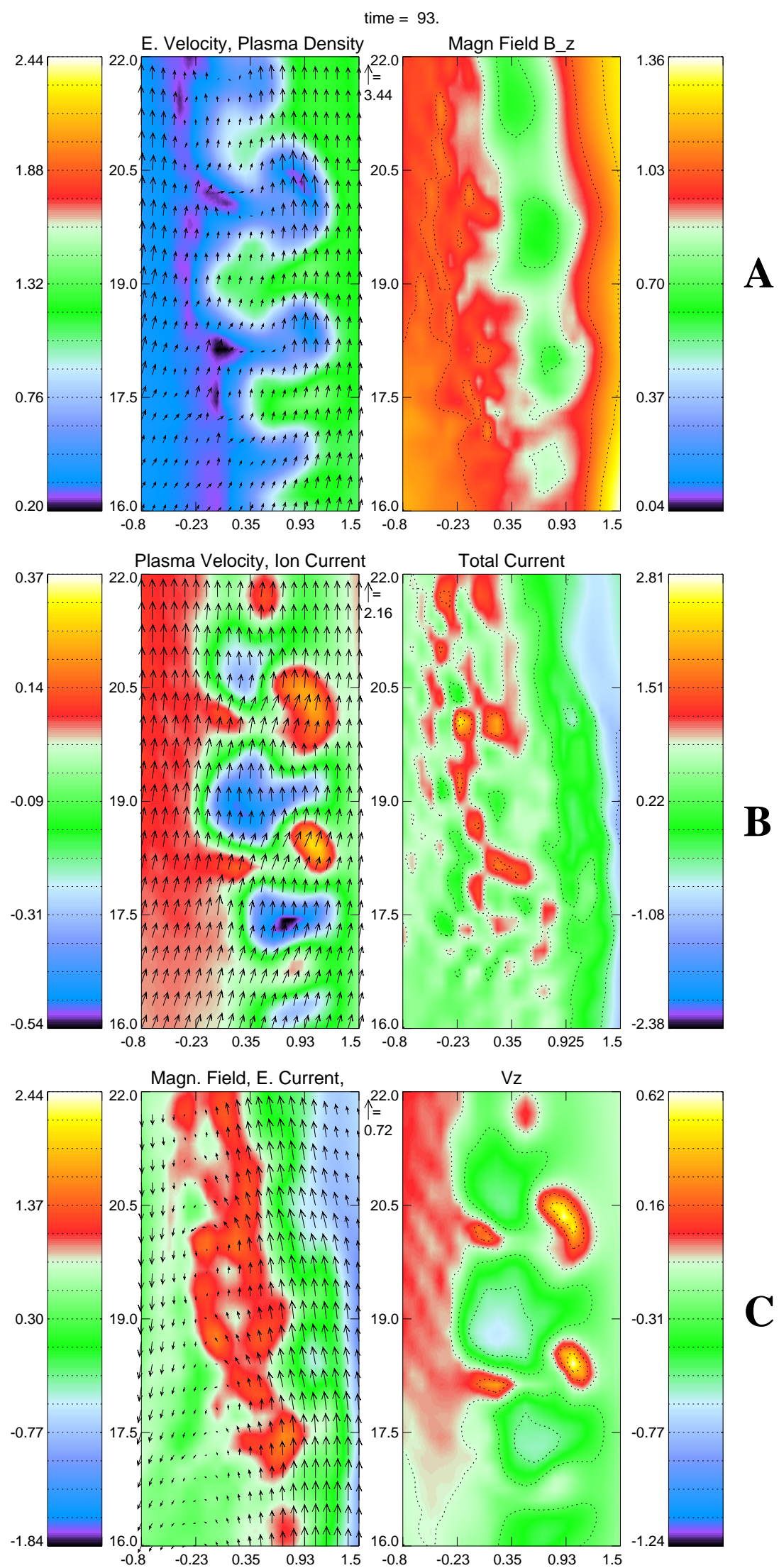

Fig. 11. (A) is a magnified domain of Fig. 10A. (B) shows plasma velocity vectors (arrows), and ion current (color code) on left and total current on right. (C) depicts magnetic field vectors (arrows) and electron current (color code) on left, and $z$-component of the plasma velocity on right. 

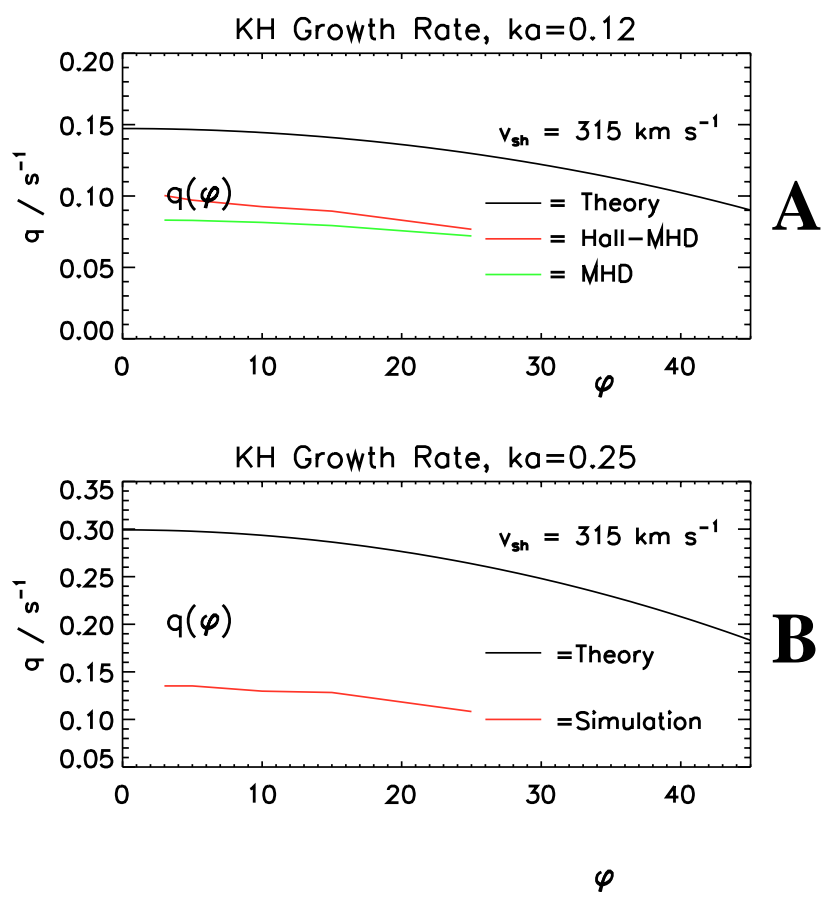

Fig. 12. (A) represents comparison of $\mathrm{KH}$ growth rates between linear MHD theory and Hall-MHD and MHD simulations for $k a=2 \pi / a \lambda=0.12$. (B) shows comparison between $\mathrm{KH}$ growth rates between theory and Hall-MHD simulations for $k a=0.25$.

(for negative vorticity case) and $14 \mathrm{~km} / \mathrm{s}$ (for positive vorticity case).

Similarly, like Thomas and Winske (1993) we find that short wavelength perturbuations grow initially, evolve into vortex structures, and eventually decay or coalesce to longer wavelength structure. We observe the ion inertia scale turbulence to be the cause for decaying of the small wavelength vortices for cases with small $\varphi$. For larger $\varphi$, mixing is also observed, but it operates mostly in the lower density regions.

There are recent results of electron inertia effects on an KH vortex by Nakumura and Fujimoto (2002), showing the formation of small-scale turbulence, however, for the case with the magnetic field entirely perpendicular, this represents the most unstable case which qualitatively agrees with the results presented here.

Simulations without $B_{z}$ indicate that reconnection occurs earlier and plasma transport is enhanced when density asymmetry across the initial shear flow layer is included.

As the Kelvin-Helmholtz vortex evolves it generates narrow layers (with thickness close to ion inertia scale) with oppositely directed magnetic fields. In these narrow layers electrons react faster to the twisting of the magnetic field forming a strong electron current. Since the magnetic field is frozen into the electron fluid, the electrons will pull the magnetic field with them as they move, generating an out-ofthe-plane magnetic field component. If the initial out-of-theplane magnetic field is zero and the density across the boundary is same, these electron motions generate a quadrupolar magnetic field structure between two reconnection points within the KH wave. When density asymmetry is included, the bipolar $B_{z}$ layers become more elongated. While the influence of the Hall term in magnetic reconnection has been studied widely, and compared with the MHD and particle simulations (Birn and Hesse, 2001; Ma and Bhattacharjee, 2001; Pritchett, 2001; Otto, 2001; Birn et al., 2001), the results presented here are the first results of a small-scale reconnection in a turbulent plasma.

While the net plasma transport due to the reconnection inside Kelvin-Helmholtz vortices is unchanged in the HallMHD approximation, the dynamical fine structure (arising from the finite ion inertia length) of the Kelvin-Helmholtz instability showed highly interesting results. Observed ion inertia scale turbulence might become very important in regions such as the Earth's magnetospheric cusps. The magnetic field in the immediate vicinity of the cusp is rather small and in the larger vicinity the geomagnetic field converges towards the cusp location such that the field close to the magnetopause rotates by $360^{\circ}$ around the cusp. Thus, there are always two regions in which the IMF is parallel or anti-parallel to the geomagnetic field, allowing reconnection to operate, and a region where the velocity component is perpendicular to the magnetic field, allowing Kelvin-Helmholtz instability to operate. Chen (1997) showed that in three dimensions a Kelvin-Helmholtz vortex can obtain filamentary structure by magnetic reconnection such that the ion inertia scale becomes important.

Acknowledgements. The research was supported by the NASA SR\&T Grant NAG-9457. In addition Katariina Nykyri's work was supported by Finnish Cultural Foundation and Finnish Academy. The computations were supported at the Arctic Region Supercomputer Center.

\section{References}

Birn, J. and Hesse, M.: GEM magnetic reconnection challenge: Resistive tearing, anisotropic pressure and Hall effects, J. Geophys. Res., 106, 3737, 2001.

Birn, J., Drake, J. F., Shay, M. A., Rogers, B. N., Denton, R. E., Hesse, M., Kuznetsova, M., Ma, Z. W., Bhattacharjee, A., Otto, A., and Pritchett, P. L.: Geospace Environment Modeling (GEM) magnetic reconnection challenge, J. Geophys. Res., 106, 3715, 2001.

Borovsky, J. E., Thomsen, M. F., and Elphic, R. C.: The driving of the plasma sheet by the solar wind, J. Geophys. Res., 103, $17617,1998$.

Brackbill, J. U. and Knoll, D. A.: Transient magnetic reconnection and unstable shear layers, Phys. Rev. Lett., 86, 2329, 2001.

Chandrasekhar, S.: Hydrodynamic and Hydromagnetic Stability, Oxford Univ. Press, New York, 1961.

Chen, Q.: Two- and three-dimensional study of the KelvinHelmholtz instability, magnetic reconnection and their mutual interaction at the magnetospheric boundary, Ph. D. Thesis, University of Alaska, Fairbanks, 1997.

Chen, Q., Otto, A., and Lee, L. C.: Tearing instability, KelvinHelmholtz instability, and magnetic reconnection, J. Geophys. Res., 102, 151, 1997. 
Fairfield, D. H., Otto, A., Mukai, T., Kokubun, S., Lepping, R. P., Steinberg, J. T., Lazarus, A. J., and Yamamoto, T.: Geotail observations of the Kelvin-Helmholtz instability at the eqatorial magnetotail boundary for parallel northward fields, J. Geophys. Res., 105, 21 159-21 174, 2000.

Fujimoto, M. and Terasawa, T.: Ion inertia effect on the KelvinHelmholtz instability, J. Geophys. Res., 96, 15 725, 1991.

Fujimoto, M. and Terasawa, T.: Anomalous ion mixing within an MHD scale Kelvin-Helmholtz vortex, J. Geophys. Res., 99, 8601, 1994.

Fujimoto, M. and Terasawa, T.: Anomalous ion mixing within an MHD scale Kelvin-Helmholtz vortex, 2, Effects of inhomogeneity, J. Geophys. Res., 100, 12 025, 1995.

Fujimoto, M., Terasawa, T., Mukai, T., Saito, Y., Yamamoto, T., and Kokubun, S.: Plasma entry from the flanks of the near-Earth magnetotail: Geotail observations, J. Geophys. Res., 103, 4391, 1998.

Huba, J. D.: Hall dynamics of the Kelvin-Helmholtz instability, Phys. Rev. Lett., 72, 2033, 1994.

Huba, J. D.: The Kelvin-Helmholtz instability: finite larmor radius magnetohydrodynamics, Geophys. Res. Lett., 23, 2907, 1996.

Keller, K. A. and Lysak, R. L.: A two-dimensional simulation of Kelvin-Helmholtz instability with magnetic shear, J. Geophys. Res., 104, 25 097, 1999.

Lee, L. C. and Olson, J. V.: Kelvin-Helmholtz instability and the variation of geomagnetic pulsation activity, Geophys. Res. Lett., 7, 777, 1980.

Ma, Z. W. and Bhattacharjee, A.: Hall magnetohydrodynamic reconnection: The Geospace Environment Modeling (GEM) challenge, J. Geophys. Res., 106, 2001.

Miura, A.: Anomalous transport by magnetohydrodynamic KelvinHelmholtz instabilities in the solar wind magnetosphere interaction, J. Geophys. Res., 89, 801, 1984.

Miura, A.: Simulation of the Kelvin-Helmholtz instability at the magnetospheric boundary, J. Geophys. Res., 92, 3195, 1987.

Miura, A.: Kelvin-Helmholtz instability at the magnetospheric boundary: Dependence on the magnetosheath sonic Mach number, J. Geophys. Res., 97, 10 655, 1992.

Miura, A. and Pritchett, P. L.: Nonlocal stability analysis of the MHD Kelvin-Helmholtz instability in a compressible plasma, J. Geophys. Res., 87, 7431, 1982.

Nakumura, T. and Fujimoto, M.: Electron inertia effects in an MHD scale Kelvin-Helmholtz vortex, Eos Trans. AGU, 83(47), Fall Meet. Suppl., Abstract, SM11B-0435, 2002.
Nikutowski, B., Büchner, J., Otto, A., Kistler, L. M., Mouikis, C., Haerendel, G., and Baumjohann, W.: Equator-S observations of reconnection coupled to surface waves, Adv. Space Res., 29(7), 1129-1134, 2002.

Nykyri, K.: Influence of the Kelvin-Helmholtz instability on the plasma transport at the magnetospheric boundary, $\mathrm{Ph}$. D. Thesis, University of Alaska, 2002.

Nykyri, K. and Otto, A.: Plasma transport at the magnetospheric boundary due to reconnection in Kelvin-Helmholtz vortices, Geophys. Res. Lett., 28, 3565-3568, 2001.

Nykyri, K., Otto, A., J. Büchner, Nikutowski, B., Baumjohann, W., Kistler, L. M., and Mouikis, C.: Equator-S observations of boundary signatures: FTE's or Kelvin-Helmholtz waves?, Geophysical Monograph 133, Earth's Low Latitude Boundary Layer, 205, 2003.

Ohtani, S., Rostoker, G., Takahashi, K., Angelopoulos, V., Nakamura, M., Waters, C., Singer, H., Kokobun, S., Tsuruda, K., Hughes, W. J., Potemra, T. A., Zanetti, L. J., Gary, J. B., Lui, A. T. Y., and Williams, D. J.: Coordinated ISTP satellite and ground observations of morningside Pc5 waves, J. Geophys. Res., 104, 2381, 1999.

Olson, J. V.: ULF signatures of polar cusp, J. Geophys. Res., 91, $10055,1986$.

Olson, J. V. and Rostoker, G.: Longitudinal phase variations of $P_{c}$ 4-5 micropulsations, J. Geophys. Res., 83, 2481, 1978.

Otto, A.: 3-D resistive MHD computations of magnetospheric physics, Comput. Phys. Commun., 59, 185, 1990.

Otto, A.: Geospace Environment Modeling (GEM) magnetic reconnection challenge: MHD and Hall MHD-constant and current dependent resistivity models, J. Geophys. Res., 106, 3751-3758, 2001.

Otto, A. and Fairfield, D. H.: Kelvin-Helmholtz instability at the magnetotail boundary: MHD simulation and comparison with Geotail observations, J. Geophys. Res., 105, 21 175, 2000.

Potter, D. E.: Computational Physics, John Wiley, New York, 1973.

Pritchett, P. L.: Geospace Environment Modeling (GEM) magnetic reconnection challenge: Simulations with a full particle electromagnetic code, J. Geophys. Res., 106, 3783, 2001.

Terasawa, T., Fujimoto, M., Karimabadi, H., and Omidi, N.: Anomalous ion mixing within a Kelvin-Helmholtz vortex in a collisionless plasma, Phys. Rev. Lett., 68, 2778, 1992.

Thomas, V. A.: Three-dimensional kinetic simulation of the KelvinHelmholtz instability, J. Geophys. Res., 100, 19429, 1995.

Thomas, V. A. and Winske, D.: Kinetic simulations of the KelvinHelmholtz instability at the magnetopause, J. Geophys. Res., 98, $11425,1993$. 